\title{
Radiation Dose from Computed Tomography Scans for Korean Pediatric and Adult Patients
}

\author{
Tristan Won', Ae-Kyoung Lee ${ }^{2}$, Hyung-do Choi ${ }^{2}$, Choonsik Lee ${ }^{3}$ \\ ${ }^{1}$ Winston Churchill High School, Potomac, MD, USA; ${ }^{2}$ Radio \& Satellite Research Division, Electronics and Telecommunications Research Institute, Daejeon, \\ Korea; ${ }^{3}$ Division of Cancer Epidemiology and Genetics, National Cancer Institute, National Institutes of Health, Rockville, MD, USA
}

\section{Original Research}

Received January 6, 2021

Revision February 18, 2021

Accepted March 3, 2021

Corresponding author: Choonsik Lee

Division of Cancer Epidemiology and Genetics, National Cancer Institute, National Institutes of Health, 9609

Medical Center Dr, Rockville, MD, USA

E-mail: choonsik.lee@nih.gov

(iD https://orcid.org/0000-0002-6219-6622

This is an open-access article distributed under the terms of the Creative Commons Attribution License (http://creativecommons.org/licenses/by-nc/4.0), which permits unrestricted use, distribution, and reproduction in any medium, provided the original work is properly cited.

Copyright $\odot 2021$ The Korean Association for Radiation Protection

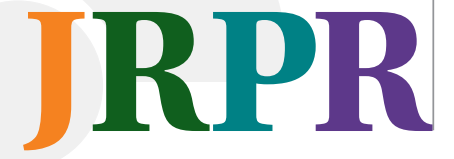

Background: In recent events of the coronavirus disease 2019 (COVID-19) pandemic, computed tomography (CT) scans are being globally used as a complement to the reverse-transcription polymerase chain reaction (RT-PCR) tests. It will be important to be aware of major organ dose levels, which are more relevant quantity to derive potential long-term adverse effect, for Korean pediatric and adult patients undergoing CT for COVID-19.

Materials and Methods: We calculated organ dose conversion coefficients for Korean pediatric and adult CT patients directly from Korean pediatric and adult computational phantoms combined with Monte Carlo radiation transport techniques. We then estimated major organ doses delivered to the Korean child and adult patients undergoing CT for COVID-19 combining the dose conversion coefficients and the international survey data. We also compared our Korean dose conversion coefficients with those from Caucasian reference pediatric and adult phantoms.

Results and Discussion: Based on the dose conversion coefficients we established in this study and the international survey data of COVID-19-related CT scans, we found that Korean 7-yearold child and adult males may receive about 4-32 mGy and 3-21 mGy of lung dose, respectively. We learned that the lung dose conversion coefficient for the Korean child phantom was up to 1.5 -fold greater than that for the Korean adult phantom. We also found no substantial difference in dose conversion coefficients between Korean and Caucasian phantoms.

Conclusion: We estimated radiation dose delivered to the Korean child and adult phantoms undergoing COVID-19-related CT examinations. The dose conversion coefficients derived for different CT scan types can be also used universally for other dosimetry studies concerning Korean CT scans. We also confirmed that the Caucasian-based CT organ dose calculation tools may be used for the Korean population with reasonable accuracy.

Keywords: Computed Tomography, Korean, Monte Carlo, Phantom, Organ Dose

\section{Introduction}

Computed tomography (CT) scans use a combination of X-ray images from multiple angles and computer processing to produce cross-sectional images of the human body. It is one of the most popular diagnostic modalities in modern medicine of which benefit is invaluable compared to its potential risk from ionizing radiation. In recent events of the coronavirus disease 2019 (COVID-19) pandemic, CT scans are being used as a complement to the reverse-transcription polymerase chain reaction (RT-PCR) tests by helping fast triage of patients while waiting for lab tests. Reports from China [1] and South Korea [2] reported a high sensitivity of chest CT scans for diagnosis of COVID-19. 
Although low dose scanning protocol has been proposed for COVID-19 [3-5], Homayounieh et al. [6] reported a substantial variation in CT protocols and radiation dose in COVID-19 patients across 62 healthcare sites from 34 countries worldwide, highlighting up to eight-fold variations (2-17 mGy) in median volumetric CT dose index (CTDI ${ }_{\text {vol }}$ ) and 10 -fold variations (76-786 $\mathrm{mGy} \cdot \mathrm{cm})$ in median dose-length product (DLP). It will be important to estimate major organ doses, which are more relevant quantity to derive potential longterm adverse effect, for Korean pediatric and adult patients undergoing CT for COVID-19.

Several studies report methods to estimate organ dose delivered to a human body when CT examinations are administered [7-11]. Most of them are based on two methods: computer simulation of CT examinations and human computational phantoms, and direct radiation dose measurements using a mannequin-like anatomy surrogate, called physical human phantoms combined with radiation sensors. Simulation-based dose data are implemented into computer programs which enable users to conveniently estimate organ dose for CT patients. However, most data are derived from the anatomical phantoms of Caucasian population. It is not clear that the Caucasian-based CT dose is also valid for Korean population. Some anatomical differences between Korean and Caucasian populations were previously reported [12-14]. Impact of the difference in body size between the two populations on CT dose descriptors was also reported [15]. To our knowledge, however, little data is available on the organ dose received by Korean pediatric and adult CT patients and its comparison with those from Caucasian populations.

The current study was intended to calculate organ dose conversion coefficients for Korean pediatric and adult CT patients directly from the Korean pediatric and adult computational phantoms combined with Monte Carlo radiation transport techniques. We then estimated major organ doses delivered to the Korean child and adult patients undergoing CT for COVID-19 combining the dose conversion coefficients and the international survey data [6]. We also compared our Korean dose conversion coefficients with those from Caucasian reference pediatric and adult phantoms.

\section{Materials and Methods}

\section{Korean Computational Human Phantoms}

We adopted two Korean computational phantoms representing a child [16] and adult [17]. The phantoms were de- veloped by researchers at the Electronics and Telecommunications Research Institute (ETRI) in South Korea using magnetic resonance image sets of a 7-year-old child male $(26 \mathrm{~kg}$ and $122 \mathrm{~cm}$ high) and a 21-year-old adult male (67 kg and $176 \mathrm{~cm}$ high) for dosimetry applications in non-ionizing radiation exposures. The child phantom is the only Korean pediatric computational phantom reported to date. The phantoms were composed of three-dimensional cubes, called voxels, stacked in the direction of $\mathrm{X}, \mathrm{Y}$, and $\mathrm{Z}$ coordinates. The number of voxels stacked in $\mathrm{X}, \mathrm{Y}$, and $\mathrm{Z}$ directions was 372 , 229 , and 408, respectively, for the Korean child phantom, and 167,87 , and 613 , respectively, for the Korean adult phantom. The volume of a single voxel in the Korean child and adult phantoms was $0.1 \times 0.1 \times 0.3 \mathrm{~cm}^{3}$ and $0.3 \times 0.3 \times 0.3 \mathrm{~cm}^{3}$, respectively. We adopted the elemental composition and density data for the organs and tissues of the two phantoms from the International Commission on Radiological Protection (ICRP) Publication 89 [18].

To more realistically simulate chest CT examinations where most patients raise their arms for torso CT scans and lower their arms for head scans, we removed the arms of the Korean models by using the image editing computer software, Image (National Institutes of Health, Bethesda, MD, USA). The voxels containing the arm structures were manually deleted slice by slice leaving only the humerus regions which are still included in chest CT scans even in arm raised posture. Fig. 1 shows the frontal views of the Korean child and adult models before and after the arm removal process.

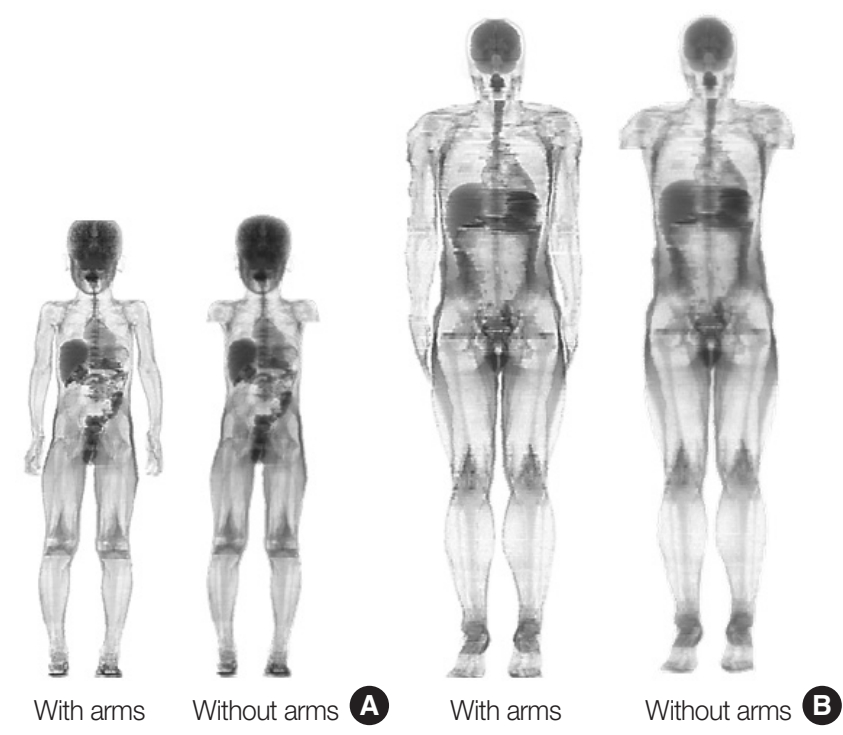

Fig. 1. Frontal views of (A) Korean child and (B) adult phantoms before (left) and after (right) the arms were removed. 


\section{Organ Dose Calculation}

We used a previously-published computer simulation model of the X-ray emitted from CT machines [19], which was experimentally validated in three independent studies [20-22]. The detailed technical features of a reference CT scanner (Siemens Sensation 16; Siemens, Erlangen, Germany) such as X-ray energy, fan beam angle, and bow-tie filter were implemented into simulations using the data obtained from the manufacturer. To conduct an accurate comparison between child and adult and between Korean and Caucasian phantoms, we consistently used a single tube potential, $120 \mathrm{kVp}$. We assumed that both pediatric and adult head scans use head X-ray filters and body scans use body X-ray filters. The simulation model of CT X-ray was combined with the Korean computational phantoms mentioned above to calculate organ dose. Our organ dose calculation for CT patients included three steps as follows: calculation of CT slicespecific organ dose conversion coefficients, derivation of CT scan type-specific organ dose conversion coefficients, and estimation of organ dose for patients undergoing CT for COVID-19. These three steps are described below in detail.

First, we calculated the amount of radiation delivered to all organs by irradiating $1 \mathrm{~cm}$ thick slice from the top of the head to the bottom of the feet in each phantom. The computer simulation was conducted using a Monte Carlo radiation transport program, called MCNPX version 2.7 [23]. MCNPX was run in the Terminal program on a MacBook (MacBook Pro 13 inch, 2017) with the CPU of $2.3 \mathrm{GHz}$ Dual-Core Intel Core i5 and 8 GB RAM. Each simulation per $1 \mathrm{~cm}$-thick slice took approximately 5 minutes. With 122 child input files $(1 \mathrm{~cm}$ thick slice for the child phantom with the height of $122 \mathrm{~cm}$ ) and 176 adult input files, completing all the simulations took around 50 hours. We simulated 10 million particle histories for each slice to reach the relative error less than $1 \%$ for major organs fully included in scan ranges (e.g., brain dose from head scan). From the Monte Carlo radiation transport, we calculated organ dose conversion coefficients, organ dose (Gy) per unit CTDI $\mathrm{vol}_{\mathrm{vol}}$ (Gy), where users can obtain organ absorbed dose (Gy) by multiplying the dose conversion coefficients to their own $\mathrm{CTDI}_{\mathrm{vol}}(\mathrm{Gy})$. By normalizing organ dose by $\mathrm{CTDI}_{\mathrm{vol}}$, the organ dose conversion coefficients become independent to CT scanner models [24]. We used CTDI ${ }_{\text {vol }}$ for $16 \mathrm{~cm}$ head CTDI phantom for head scan and that for $32 \mathrm{~cm}$ body CTDI phantom for other torso scans for normalization.

Second, we derived CT scan type-specific organ dose con- version coefficients. We summed up the organ dose conversion coefficients within the slice range covering the different parts of the phantoms representing head, chest, abdomenpelvis (AP), chest-abdomen-pelvis (CAP), and head-chestabdomen-pelvis (HCAP) scans. Although only chest scans are conducted for the diagnosis of COVID-19, we also included other scan ranges such as head, AP, CAP, and HCAP in case chest scan may cover beyond the chest region. We included the following organs in the calculation: brain, thyroid, lungs (left and right averaged), small intestine wall, colon wall, stomach wall, liver, kidneys (left and right averaged), urinary bladder, heart, esophagus, spleen, and gonads. The coverage of the scan was defined from the scan protocol from the National Institutes of Health (NIH) Clinical Center [19]. Head scans covered from the top of the head to the second cervical vertebra. Chest scans ranged from the top part of the clavicles to the mid-level of the liver. AP scans started from the top of the liver down to the middle of the upper femoral heads. CAP scans combined the scan ranges of chest and AP scans and HCAP scans combined the scan ranges of head and CAP scans. The distance of each scan coverage was obtained from the phantom images using ImageJ.

Third, we estimated major organ doses delivered to Korean pediatric and adult patients undergoing COVID-19-related CT scans. Since Korean data were not included in the 34 surveyed countries reported by Homayounieh et al. [6], we had to assume that similar level of CTDI $_{\text {vol }}$ was used in Korea for the diagnosis and follow-up of COVID-19. We estimated organ dose for Korean pediatric and adult patients by multiplying the organ dose conversion coefficients for different scan types derived from the previous step with the $\mathrm{CTDI}_{\mathrm{vol}}$ values used for COVID-19 reported in the international survey.

\section{Comparison of Dose Conversion Coefficients with Caucasian Data}

We compared the resulting organ doses from the two Korean phantoms with those from Caucasian reference phantoms published from the ICRP $[25,26]$. To calculate organ dose for the ICRP Caucasian phantoms, we used a previously-published computer program for CT dose calculation, $\mathrm{Na}$ tional Cancer Institute dosimetry system for CT (NCICT) [19], which is based on the organ dose database calculated from the ICRP pediatric and adult phantoms. The program uses the identical X-ray source spectra to that used in the current study. We compared organ doses from the Korean child phan- 
Table 1. Organ Absorbed Dose per CTDlvol (mGy/mGy) for the Korean Child and Adult Phantoms Undergoing Head, Chest, AP, CAP, and HCAP Examinations

\begin{tabular}{|c|c|c|c|c|c|c|c|c|c|c|}
\hline \multirow{3}{*}{ Organs } & \multicolumn{10}{|c|}{ CTDlvol (mGy/mGy) } \\
\hline & \multicolumn{5}{|c|}{ Korean 7-year-old male } & \multicolumn{5}{|c|}{ Korean adult male } \\
\hline & Head & Chest & AP & CAP & HCAP & Head & Chest & AP & CAP & HCAP \\
\hline Brain & 0.843 & 0.015 & 0.003 & 0.016 & 1.859 & 0.839 & 0.011 & 0.001 & 0.014 & 1.767 \\
\hline Thyroid & 0.009 & 1.664 & 0.063 & 1.681 & 2.432 & 0.062 & 1.056 & 0.004 & 1.058 & 2.227 \\
\hline Lungs & 0.004 & 1.894 & 0.678 & 1.988 & 2.059 & 0.004 & 1.254 & 0.185 & 1.290 & 1.314 \\
\hline SI wall & 0.000 & 0.086 & 1.881 & 1.900 & 1.903 & 0.000 & 0.079 & 1.426 & 1.444 & 1.444 \\
\hline Colon wall & 0.000 & 0.073 & 1.675 & 1.690 & 1.693 & 0.000 & 0.039 & 1.364 & 1.373 & 1.373 \\
\hline Stomach wall & 0.001 & 0.948 & 1.741 & 1.885 & 1.899 & 0.000 & 0.757 & 1.251 & 1.405 & 1.407 \\
\hline Liver & 0.001 & 1.104 & 1.732 & 1.886 & 1.903 & 0.000 & 0.971 & 1.163 & 1.405 & 1.407 \\
\hline Kidneys & 0.001 & 0.378 & 1.734 & 1.798 & 1.806 & 0.000 & 0.191 & 1.406 & 1.448 & 1.448 \\
\hline Urinary bladder & 0.000 & 0.005 & 1.357 & 1.359 & 1.359 & 0.000 & 0.006 & 0.815 & 0.816 & 0.816 \\
\hline Heart & 0.002 & 1.881 & 0.918 & 2.001 & 2.046 & 0.002 & 1.217 & 0.369 & 1.284 & 1.293 \\
\hline Esophagus & 0.003 & 1.698 & 0.422 & 1.763 & 1.896 & 0.012 & 0.909 & 0.419 & 1.190 & 1.327 \\
\hline Spleen & 0.001 & 0.754 & 1.725 & 1.818 & 1.829 & 0.000 & 0.818 & 1.153 & 1.274 & 1.275 \\
\hline Gonads & 0.000 & 0.002 & 0.106 & 0.107 & 0.107 & 0.000 & 0.001 & 0.215 & 0.215 & 0.216 \\
\hline Total skeleton & 0.001 & 0.090 & 0.040 & 0.127 & 0.132 & 0.002 & 0.090 & 0.177 & 0.267 & 0.272 \\
\hline
\end{tabular}

CTDIvol, volumetric computed tomography dose index; AP, abdomen-pelvis; CAP, chest-abdomen-pelvis; HCAP, head-chest-abdomen-pelvis.

tom with those from 5- and 10-year-old Caucasian reference phantoms. In addition, we compared organ doses from the Korean adult male phantom with those from the Caucasian adult male phantom.

To better analyze organ dose differences across the different computational phantoms, we measured the representative diameter of phantoms, called effective diameter, at the middle level within a given scan range for the head, chest, AP, and CAP scans. Effective diameter is defined as a geometric mean of the width and depth of the body on a single crosssectional CT image [27].

\section{Results and Discussion}

\section{Organ Dose Coefficients for Korean Child and Adult Patients}

We found that the Korean child phantom received a greater absorbed dose in most scans compared to the adult phantom at the same CTDI ${ }_{\text {vol }}$ (Table 1). The lungs (chest scan), and kidneys (AP scan) of the Korean child phantom received 1.5- and 1.2-fold greater dose than those of the adult phantom, respectively. The brain dose of the child phantom, 0.843 $\mathrm{mGy} / \mathrm{mGy}$, was slightly greater than that of the adult phantom, $0.839 \mathrm{mGy} / \mathrm{mGy}$. Although this is true for most cases, in some examples, the adult phantom received a greater absorbed dose than the child phantom: the thyroid dose in head scan, 0.009 (child) versus 0.062 (adult) mGy/mGy.
Table 2. Effective Diameter Measured from Korean and Caucasian Computational Phantoms for the Head, Chest, AP, and CAP Scans

\begin{tabular}{llcccc}
\hline \multirow{2}{*}{$\begin{array}{c}\text { Age } \\
\text { group }\end{array}$} & \multirow{2}{*}{ Phantoms } & \multicolumn{4}{c}{ Effective diameter $(\mathrm{cm})$} \\
\cline { 3 - 6 } & & Head & Chest & AP & CAP \\
\hline \multirow{2}{*}{ Child } & Korean 7-year-old male & 15 & 18 & 17 & 18 \\
& Caucasian 5-year-old male & 16 & 19 & 16 & 18 \\
& Caucasian 10-year-old male & 16 & 25 & 21 & 22 \\
\multirow{3}{*}{ Adult } & Korean adult male & 17 & 28 & 24 & 25 \\
& Caucasian adult male & 20 & 28 & 26 & 26 \\
\hline
\end{tabular}

AP, abdomen-pelvis; CAP, chest-abdomen-pelvis.

The Korean child phantom showed overall greater organ dose compared to the Korean adult phantom. This can be explained by the effective diameters measured from the two phantoms as shown in Table 2. The effective diameter of the Korean child phantom at the head, chest, and AP levels is smaller than that of the Korean adult phantom by $12 \%, 35 \%$, and $29 \%$, respectively. CT X-rays enter the human body and experience attenuation before reaching a given organ contributing to an organ dose. Therefore, the organ absorbed dose is inversely proportional to the amount of the human tissue that X-rays penetrate, which is reflected in the effective diameter of the human body. The effective diameter of the Korean child phantom in chest CT scan, $18 \mathrm{~cm}$, is much smaller than that of the adult phantom, $28 \mathrm{~cm}$. As described in the results section, the lungs of the child phantom received 1.5fold greater dose than that of the adult phantom in a chest 
CT scan. The greater organ doses in the child phantom compared to the adult can be explained by the smaller effective diameter in the child compared to the adult phantom in other scans such as the head, AP, and CAP scans.

The organs receiving the greatest dose differed between the Korean child and adult phantoms (Table 1). The heart and kidneys, followed by the kidney and small intestine wall, received the highest dose in CAP scans for the child and adult phantoms, respectively. Organ dose in CT depends on how deep a certain organ is located under the body surface, which is largely affected by individual variability. It seems that the heart is located shallower than the kidneys in the Korean child phantom, whereas the depth from the body surface of the kidneys is shallower than the small intestine wall in the adult phantom.

Organs outside the scan coverages also received measurable doses from scattered X-ray. The small intestine of the child and adult phantoms received 0.086 and $0.079 \mathrm{mGy} / \mathrm{mGy}$ in chest scans even though the organ is outside the chest scan coverage, from the top of the clavicles to the middle location of the liver. The brain also received some doses $(0.016 \mathrm{mGy} /$ $\mathrm{mGy}$ in the child and $0.100 \mathrm{mGy} / \mathrm{mGy}$ in the adult) from CAP scans which do not include any part of the brain.

\section{Organ Doses for Korean Patients Undergoing COVID-19-Related CT}

Assuming the similar level of CTDI vol $_{\text {( }}(2-17 \mathrm{mGy})$ reported from 34 countries [6] were used for COVID-19 in Korea for both pediatric and adult patients, Korean 7-year-old child and adult males may receive about 4-32 mGy and 3-21 mGy of lung dose, respectively, which were derived using the range of CTDI ${ }_{\mathrm{vol}}$ (2-17 mGy) and the lung dose conversion coefficients for chest CT scans, $1.894 \mathrm{mGy} / \mathrm{mGy}$ for child and 1.254 $\mathrm{mGy} / \mathrm{mGy}$ for adult (Table 1). If longer anatomical regions than chest may be scanned, which may be close to HCAP scans, the lung dose conversion coefficients of $2.059 \mathrm{mGy} /$ $\mathrm{mGy}$ (child) and $1.314 \mathrm{mGy} / \mathrm{mGy}$ (adult) can be used for calculations. The resulting lung doses would be 4-35 mGy and 3-22 mGy, which are slightly greater than those from chest scans.
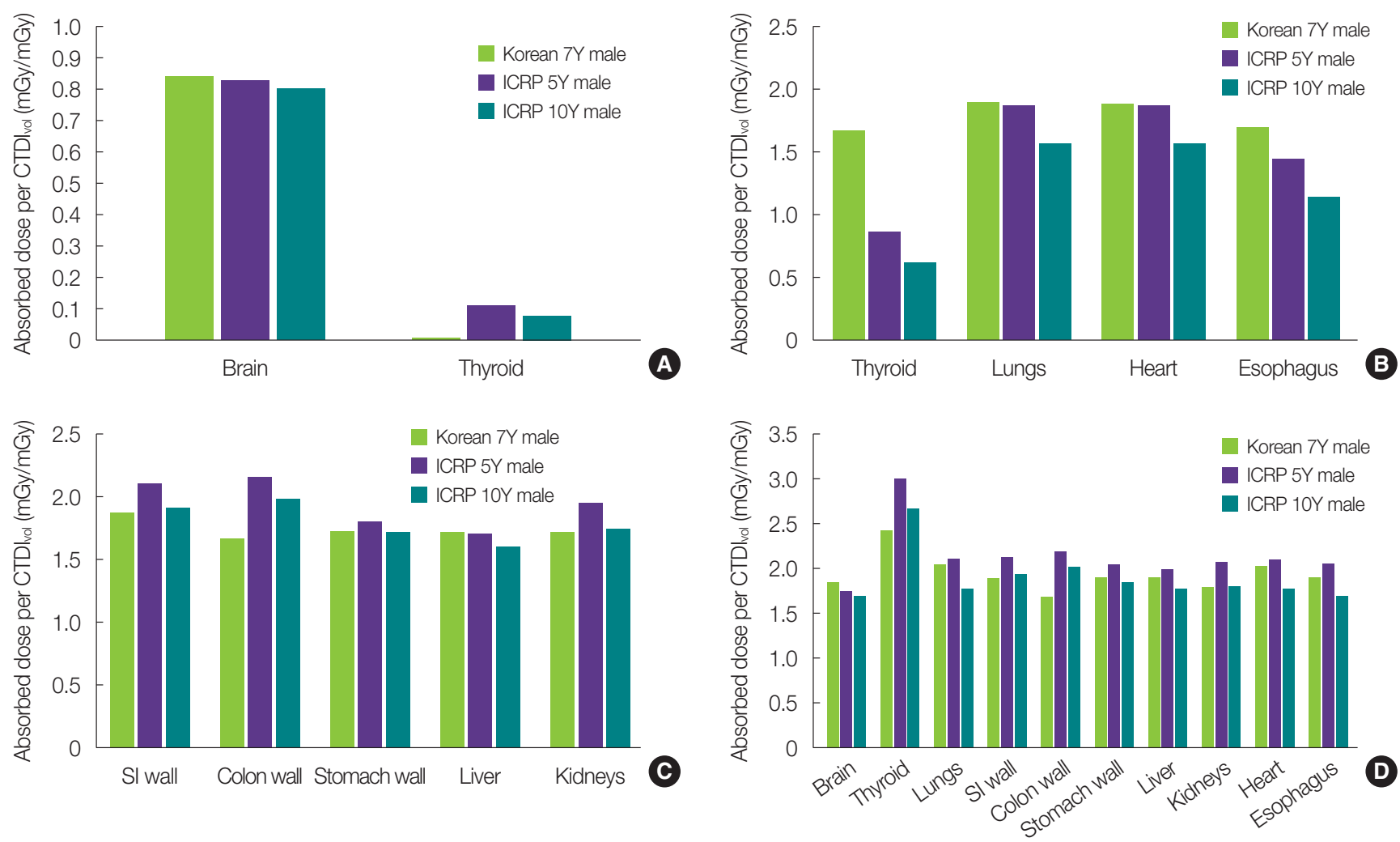

Fig. 2. Comparison of organ absorbed dose per unit CTDIvol (mGy/mGy) from the Korean 7-year-old male phantom with those from the Caucasian reference ICRP 5- and 10-year-old male phantoms for (A) head, (B) chest, (C) abdomen-pelvis, and (D) head-chest-abdominal-pelvis examinations. CTDlvol, volumetric computed tomography dose index; ICRP, International Commission on Radiological Protection. 


\section{Comparison of Organ Dose Conversion Coefficients with Caucasian Data}

In comparison of dose conversion coefficients between the Korean and ICRP phantoms, we found that the Korean child phantom (Fig. 2) received slightly greater dose than ICRP 5- and 10-year-old phantoms in head and chest scans, whereas smaller dose than ICRP 5-year-old but close to ICRP 10-year-old in AP and CAP scans. From the results, we can infer that the head and chest circumferences of the Korean 7-year-old phantom are smaller than those of the ICRP 5and 10-year-old phantoms, whereas the abdominal circumference of the Korean 7-year-old is greater than ICRP 5-yearold but similar to ICRP 10-year-old. The Korean adult phantom (Fig. 3) overall received slightly smaller dose than the ICRP adult male phantom except head scans where the Korean phantom showed greater dose than the ICRP phantom. The brain of the Korean adult male phantom received about $16 \%$ greater dose than that of the ICRP adult male (Fig. 3A). We can again infer that the circumference of the Korean adult phantom is smaller than that of the ICRP adult phantom for all body parts except head. These interpretations can
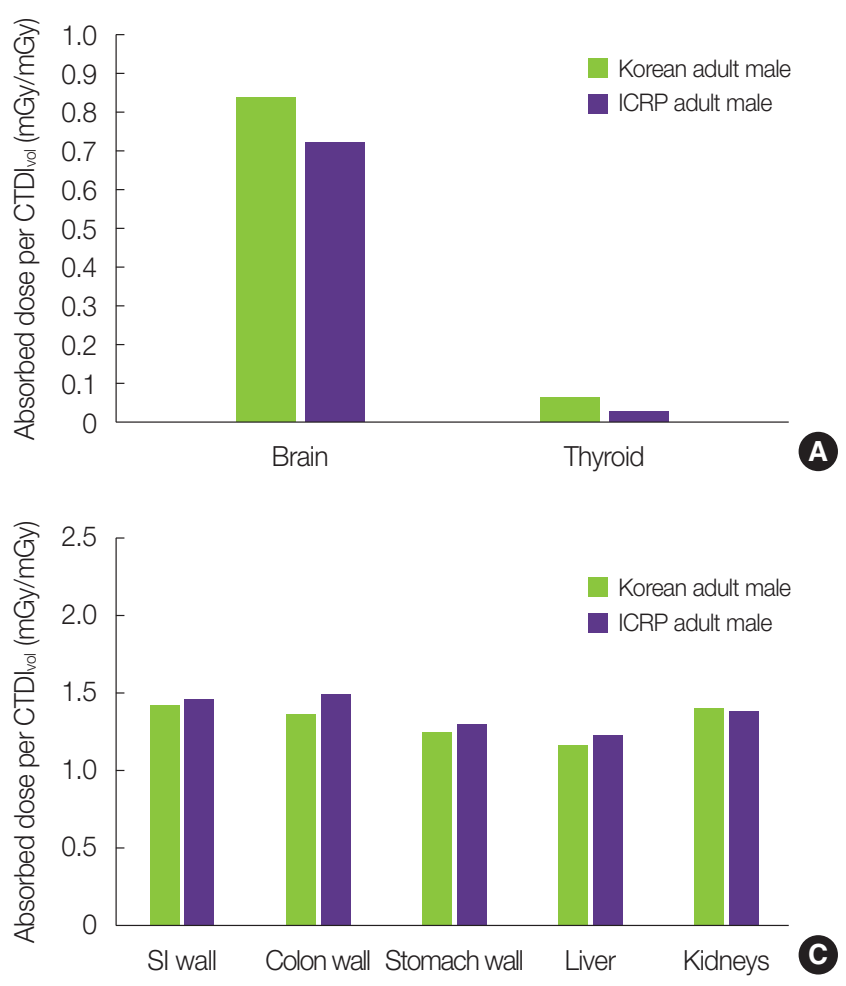

be confirmed by the effective diameters measured from the Korean and Caucasian phantoms (Table 2).

Thyroid dose substantially varied across different phantoms in head and chest scans. The thyroid of the Korean child phantom (0.009 $\mathrm{mGy} / \mathrm{mGy}$ ) received only about $8 \%$ of the dose delivered to the thyroid of the ICRP 5-year-old phantom $(0.110 \mathrm{mGy} / \mathrm{mGy})$ whereas in chest scan, it received (1.664 mGy/mGy) about two times greater dose than that of the ICRP 5-year-old ( $0.860 \mathrm{mGy} / \mathrm{mGy})$. The coefficient of variation (COV), standard deviation divided by mean, among the thyroid doses of the child and adult phantoms in the head scan was $79 \%$ and $50 \%$, respectively. The COV in the thyroid doses of the child and adult in the chest scan was $52 \%$ and $31 \%$, respectively. Compared to these COVs, the stomach dose in AP scans, for example, was $3 \%$ for both the child and adult phantoms. This inconsistency could be attributed to the location of the thyroid within the human anatomy. For example, the thyroid of the Korean child phantom may be lower than the ICRP pediatric phantoms so that a smaller portion of the thyroid is included in the head scan, but a greater portion is in the chest scan coverage. This analysis is confirmed
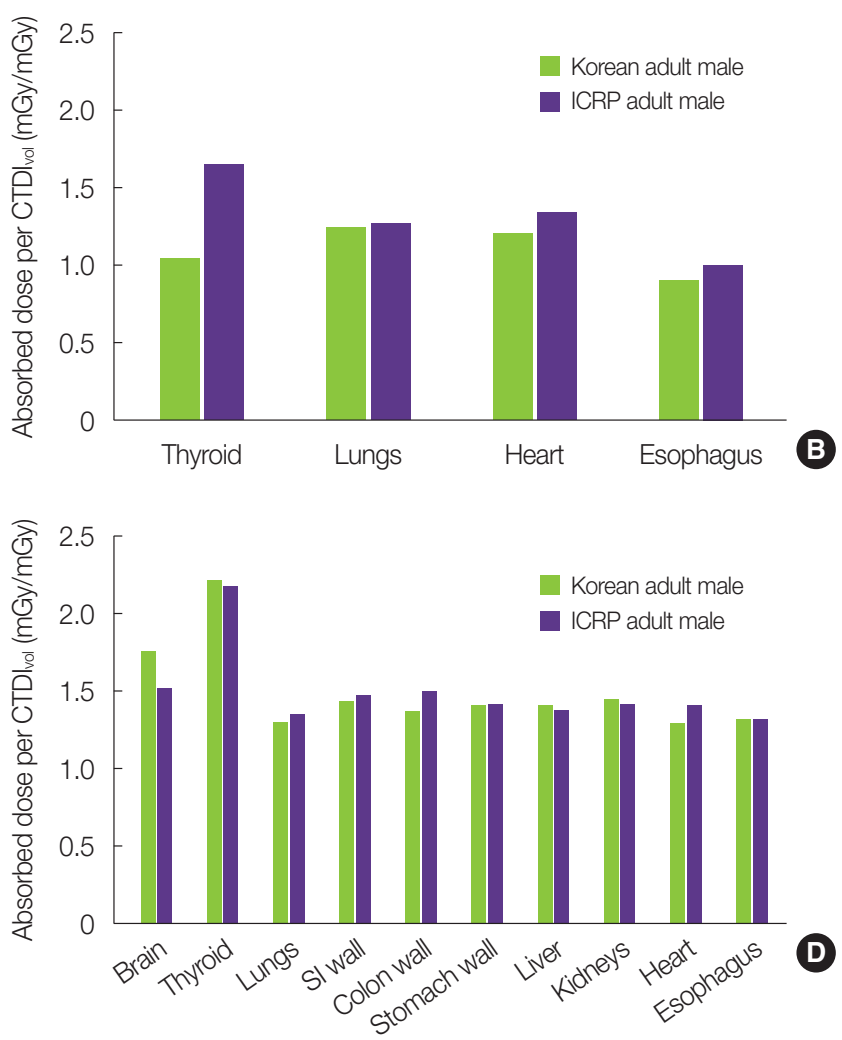

Fig. 3. Comparison of organ absorbed dose per unit CTDIvo (mGy/mGy) from the Korean adult male phantom with those from the Caucasian reference ICRP adult male phantoms for (A) head, (B) chest, (C) abdomen-pelvis, and (D) head-chest-abdominal-pelvis examinations. CTDlvol, volumetric computed tomography dose index; ICRP, International Commission on Radiological Protection. 
in the thyroid dose comparison in HCAP scan (Figs. 2D and 3D). In the HCAP scans, the full thyroid is included in the scan coverage, so the vertical location of the thyroid does not affect its dose. Substantial difference in the thyroid between the Korean and ICRP phantoms observed in head and chest scans do not exist anymore. We learned that the Caucasianbased CT dose calculator can be used for the Korean pediatric and adult patients with reasonable accuracy.

\section{Limitations}

We are aware that the current study still has the following limitations. First, we only used two Korean computational phantoms representing a child and adult, which may make it difficult to generalize the findings from this study to the whole Korean population. However, to our knowledge, the child phantom developed by the ETRI researchers is the only pediatric Korean computational phantom. We plan to extend the current study to include more Korean adult male and female phantoms into organ dose calculations. Second, we used the international survey data for COVID-19 CT dose calculations because Korean-specific data were not available. Nationwide survey is required to obtain more accurate range of CT dose descriptors. Third, we did not account for tube current modulation techniques in our organ dose calculations. If properly applied, the technique is reported to reduce some organ doses such as the lungs (about 14\%-17\%) in chest scan $[19,28]$. We are exploring a prospective method to create a tube current modulation profile from the voxel phantoms by using ray-tracing algorithms [29], which then can be implemented into dose conversion coefficients. Fourth, we are aware that the volume of a single voxel in the Korean adult phantom $\left(0.027 \mathrm{~cm}^{3}\right)$ is about nine-times greater than that of the Korean child phantom $\left(0.003 \mathrm{~cm}^{3}\right)$. Our previous study [30] confirms that the finer voxel resolution would provide the more accurate dose but the error from a resolution $<25 \mathrm{~mm}^{3}$ (the Korean adult phantom) is less than $1 \%-2 \%$. Lastly, we calculated organ dose coefficients only for the tube potential of $120 \mathrm{kVp}$ for both the child and adult phantoms. Because both organ dose and $\mathrm{CTDI}_{\mathrm{vol}}$ include the impact of tube potential, however, the resulting organ dose coefficients, organ dose normalized to $\mathrm{CTDI}_{\mathrm{vol}}$, would not change much by the change in tube potential.

\section{Conclusion}

We estimated radiation dose delivered to the Korean child and adult phantoms undergoing COVID-19-related CT examinations by using Korean computational phantom-based organ dose conversion coefficients and the international survey data of CT dose descriptors. The dose conversion coefficients derived for different CT scan types can be also used universally for other dosimetry studies concerning Korean CT scans. We also confirmed that the Caucasian-based CT organ dose calculation tools may be used for the Korean population with reasonable accuracy.

\section{Conflict of Interest}

No potential conflict of interest relevant to this article was reported.

\section{Acknowledgements}

This research was partially funded by the MSIP (Ministry of Science, ICT and Future Planning), Korea in the ICT R\&D Program 2013, and by the intramural research program of the National Institutes of Health, National Cancer Institute, Division of Cancer Epidemiology and Genetics. The contents are solely the responsibility of the authors and does not necessarily represent the official views of the National Institutes of Health.

\section{Author Contribution}

Conceptualization: Lee C. Data curation: Won T, Lee A, Choi H, Lee C. Formal analysis: Lee C. Funding acquisition: Lee C. Methodology: Won T, Lee A, Choi H, Lee C. Project administration: Lee C. Visualization: Won T, Lee C. Writing original draft: Won T, Lee C. Writing - review \& editing: Won T, Lee A, Choi H, Lee C. Investigation: Lee C. Resources: Choi H, Lee C. Software: Won T, Lee C. Supervision: Lee A, Lee C. Validation: Lee C.

\section{References}

1. Ai T, Yang Z, Hou H, Zhan C, Chen C, Lv W, et al. Correlation of chest CT and RT-PCR testing for coronavirus disease 2019 (COVID-19) in China: a report of 1014 cases. Radiology. 2020;296: E32-E40.

2. Yoon SH, Lee KH, Kim JY, Lee YK, Ko H, Kim KH, et al. Chest radiographic and CT findings of the 2019 novel coronavirus disease (COVID-19): analysis of nine patients treated in Korea. Korean J Radiol. 2020;21:494-500. 
3. Dangis A, Gieraerts C, De Bruecker Y, Janssen L, Valgaeren H, Obbels D, et al. Accuracy and reproducibility of low-dose submillisievert chest CT for the diagnosis of COVID-19. Radiol Cardiothorac Imaging. 2020;2:e200196.

4. Kang Z, Li X, Zhou S. Recommendation of low-dose CT in the detection and management of COVID-2019. Eur Radiol. 2020; 30:4356-4357.

5. Agostini A, Floridi C, Borgheresi A, Badaloni M, Esposto Pirani P, Terilli F, et al. Proposal of a low-dose, long-pitch, dual-source chest CT protocol on third-generation dual-source CT using a tin filter for spectral shaping at $100 \mathrm{kVp}$ for CoronaVirus Disease 2019 (COVID-19) patients: a feasibility study. Radiol Med. 2020; 125:365-373.

6. Homayounieh F, Holmberg O, Umairi RA, Aly S, Basevicius A, Costa PR, et al. Variations in CT utilization, protocols, and radiation doses in COVID-19 pneumonia: results from 28 countries in the IAEA study. Radiology. 2021;298:E141-E151.

7. Lee C, Kim KP, Long D, Fisher R, Tien C, Simon SL, et al. Organ doses for reference adult male and female undergoing computed tomography estimated by Monte Carlo simulations. Med Phys. 2011;38:1196-1206.

8. Lee C, Lee C, Staton RJ, Hintenlang DE, Arreola MM, Williams $\mathrm{JL}$, et al. Organ and effective doses in pediatric patients undergoing helical multislice computed tomography examination. Med Phys. 2007;34:1858-1873.

9. Ding A, Gao Y, Liu H, Caracappa PF, Long DJ, Bolch WE, et al. VirtualDose: a software for reporting organ doses from CT for adult and pediatric patients. Phys Med Biol. 2015;60:5601-5625.

10. DeMarco JJ, Cagnon CH, Cody DD, Stevens DM, McCollough CH, O'Daniel J, et al. A Monte Carlo based method to estimate radiation dose from multidetector CT (MDCT): cylindrical and anthropomorphic phantoms. Phys Med Biol. 2005;50:3989-4004.

11. Li X, Samei E, Segars WP, Sturgeon GM, Colsher JG, Frush DP. Patient-specific dose estimation for pediatric chest CT. Med Phys. 2008;35:5821-5828.

12. Park S, Lee JK, Kim JI, Lee YJ, Lim YK, Kim CS, et al. In vivo organ mass of Korean adults obtained from whole-body magnetic resonance data. Radiat Prot Dosimetry. 2006;118:275-279.

13. Choi C, Yeom YS, Nguyen TT, Lee H, Han H, Shin B, et al. Korean anatomical reference data for adults for use in radiological protection. J Korean Phys Soc. 2018;72:183-191.

14. Lee C, Lee C, Park SH, Lee JK. Development of the two Korean adult tomographic computational phantoms for organ dosimetry. Med Phys. 2006;33:380-390.

15. Kim EY, Kim TJ, Goo JM, Kim HY, Lee JW, Lee S, et al. Size-specific dose estimation in the Korean lung cancer screening project: does a 32-cm diameter phantom represent a standard-sized patient in Korean population? Korean J Radiol. 2018;19:11791186.
16. Lee AK, Byun JK, Park JS, Choi HD, Yun J. Development of 7-yearold Korean child model for computational dosimetry. ETRI J. 2009;31:237-239.

17. Lee AK, Choi WY, Chung MS, Choi HD, Choi JI. Development of Korean male body model for computational dosimetry. ETRI J. 2006;28:107-110.

18. Valentin J. Basic anatomical and physiological data for use in radiological protection: reference values: ICRP Publication 89. Ann ICRP. 2002;32:1-277.

19. Lee C, Kim KP, Bolch WE, Moroz BE, Folio L. NCICT: a computational solution to estimate organ doses for pediatric and adult patients undergoing CT scans. J Radiol Prot. 2015;35:891-909.

20. Dabin J, Mencarelli A, McMillan D, Romanyukha A, Struelens L, Lee C. Validation of calculation algorithms for organ doses in CT by measurements on a 5 year old paediatric phantom. Phys Med Biol. 2016;61:4168-4182.

21. Long DJ, Lee C, Tien C, Fisher R, Hoerner MR, Hintenlang DE, et al. Monte Carlo simulations of adult and pediatric computed tomography exams: validation studies of organ doses with physical phantoms. Med Phys. 2013;40:013901.

22. Giansante L, Martins JC, Nersissian DY, Kiers KC, Kay FU, Sawamura MV, et al. Organ doses evaluation for chest computed tomography procedures with TL dosimeters: comparison with Monte Carlo simulations. J Appl Clin Med Phys. 2019;20:308-320.

23. Pelowitz DB. MCNPX user's manual version 2.7.0. Los Alamos, NM: Los Alamos National Laboratory; 2011.

24. Turner AC, Zankl M, DeMarco JJ, Cagnon CH, Zhang D, Angel E, et al. The feasibility of a scanner-independent technique to estimate organ dose from MDCT scans: using $\mathrm{CTDI}_{\mathrm{vol}}$ to account for differences between scanners. Med Phys. 2010;37:1816-1825.

25. Bolch WE, Eckerman K, Endo A, Hunt JG, Jokisch DW, Kim CH, et al. ICRP Publication 143: paediatric reference computational phantoms. Ann ICRP. 2020;49:5-297.

26. ICRP Publication 110: adult reference computational phantoms. Ann ICRP. 2009;39:1-166.

27. Boone JM, Strauss KJ, Cody DD, McCollough CH, McNitt-Gray MF, Toth TL. Report of AAPM TG 204: size-specific dose estimates (SSDE) in pediatric and adult body CT examinations. College Park, MD: American Association of Physicians in Medicine; 2011.

28. Schlattl H, Zankl M, Becker J, Hoeschen C. Dose conversion coefficients for CT examinations of adults with automatic tube current modulation. Phys Med Biol. 2010;55:6243-6261.

29. Li X, Segars WP, Samei E. The impact on CT dose of the variability in tube current modulation technology: a theoretical investigation. Phys Med Biol. 2014;59:4525-4548.

30. Lee C, Badal A, Yeom YS, Griffin KT, McMillan D. Dosimetric impact of voxel resolutions of computational human phantoms for external photon exposure. Biomed Phys Eng Express. 2019; 5:065002. 